\title{
The Structure of Digroups
}

\author{
Catherine Crompton \\ Department of Mathematics \\ Agnes Scott College \\ $141 \mathrm{E}$. College Avenue \\ Decatur, Georgia 30030 USA \\ Linda Scalici
Department of Mathematics \\ Bucknell University \\ Moore Avenue \\ Lewisburg, Pennsylvania 17837 USA
}

Received: November 7, 2005

Accepted: August 28, 2006 \begin{abstract}
$b=b \vdash a$, but $a \vdash b \neq a \dashv b$.
\section{INTRODUCTION}

A vexing problem in the theory of Leibniz algebra is finding a generalization of Lie's third theorem. Lie's third theorem associates a (local) Lie group to any Lie algebra, real or complex. Seeking appropriate analogues of Lie groups for Leibiniz algebras, Loday [1] became so exasperated at one point that he dubbed these objects coquecigrues-absurdity incarnate. Kinyon [2] found a partial solution using digroups (defined below), as follows: if a Leibniz algebra splits over an ideal containing the ideal generated by squares, then it is isomorphic to the tangent algebra of a linear Lie digroup. In particular, if the split Leibniz algebra is actually a Lie algebra, then the linear Lie digroup coincides with the Lie group from Lie's third theorem. For Leibniz algebras that do not split, the problem remains open.
\end{abstract}

ABSTRACT

A digroup is an algebra defined on a set having two associative binary operations, $\vdash$ and $\dashv$. Digroups play an important role in an open problem in the theory of Leibniz algebras. We present a brief overview of digroups and a set of more general axioms for a digroup than used previously.

We then consider several properties of a digroup having distinct elements $a$ and $b$ such that $a \vdash$

Loday was the first to use the idea of a digroup [3]; since then others have refined Loday's work, e.g., [4] and [5]. Kinyon modified Loday's definition of digroup to give one that is much cleaner (see below). As part of his partial solution to the coquecigrue problem, he showed that every digroup is a product of a group and a trivial digroup. J. D. Phillips [6] gave an even simpler basis of axioms for a digroup. In this paper, we generalize Kinyon's first axiom of associativity to give yet a more general axiom scheme for digroups. Then we look at some properties of pairs of elements that commute under one of the binary operations in a digroup.

Though we will not make use of this definition in this paper, a Lie digroup is a smooth manifold with a digroup structure such that the digroup operations are smooth mappings. A linear Lie digroup is a Lie digroup recovered from the product of a Lie group and a module in a natural way [2]. 


\section{AXIOMIZATIONS OF DIGROUPS} follows.

Kinyon's definition of a digroup is as

Definition 1 (Kinyon). A digroup is a set, G, equipped with two binary operations, $\vdash$ and $\dashv$, with a unary operation, ${ }^{\dagger}$, and with nullary operation, 1 , satisfying each of the following six axioms:

G1. $(G, \vdash)$ and $(G, \dashv)$ are both semigroups.

G2. $(x \vdash y) \dashv z=x \vdash(y \dashv z)$.

G3. $x \dashv(y \vdash z)=x \dashv y \dashv z$.

G4. $(x \dashv y) \vdash z=x \vdash y \vdash z$.

G5. $1 \vdash x=x=x \dashv 1$.

G6. $x \vdash x^{\dagger}=1=x^{\dagger} \dashv x$.

Note that a digroup is essentially a left group and a right group together with some compatibility axioms. J. D. Phillips [6] showed that $G 2$ through $G 4$ can be simplified as follows:

Theorem 1 (Phillips). A set, $\mathrm{G}$, is a digroup, if and only if it is equipped with two binary operators, $\vdash$ and $\dashv$, with a unary operation, $\dagger$, and with nullary operation, 1 , satisfying the following four axioms:

G1. $(G, \vdash)$ and $(G, \dashv)$ are both semigroups.

$G 2^{*} x \vdash(x \dashv z)=(x \vdash x) \dashv z$.

G5. $1 \vdash x=x=x \dashv 1$.

G6. $x \vdash x^{\dagger}=1=x^{\dagger} \dashv x$.

We will show that $G 1$, the associativity of $\vdash$ and $\dashv$, may be weakened to a form of the
Semi-Moufang identity.

Theorem 2. A set, $G$, is a digroup if and only if it is closed under two binary operations $\vdash$ and $\dashv$, has a unary operation, $\dagger$, and with nullary operation, 1 , satisfying the following axioms:

G1* $\vdash$ and $\dashv$ are semi-Moufang, i.e.,

$$
\begin{aligned}
& x \vdash((y \vdash z) \vdash x)=(x \vdash y) \vdash(z \vdash x) \text { and } \\
& x \dashv((y \dashv z) \dashv x)=(x \dashv y) \dashv(z \dashv x) .
\end{aligned}
$$

G2. $(x \vdash y) \dashv z=x \vdash(y \dashv z)$.

G5. $1 \vdash x=x=x \dashv 1$.

G6. $x \vdash x^{\dagger}=1=x^{\dagger} \dashv x$.

Proof. All digroups satisfy these axioms because they are a weaker set of axioms. Conversely, let $G$ be an algebra satisfying these axioms. Using Phillips' Axioms of a digroup, we need only prove associativity of the two binary operations, (since G2 implies $G 2 *$ by setting $y=x$ ). We will show the proof for the associativity of $\vdash$; the proof for $\dashv$ is similar.

We introduce six auxiliary identities to help in proving $(x \vdash y) \vdash z=x \vdash(y \vdash z)$ :

A1. $(\mathrm{x} \vdash \mathrm{y}) \vdash 1=\mathrm{x} \vdash(\mathrm{y} \vdash 1)$.

A2. $1 \dashv \mathrm{x}=\mathrm{x} \vdash 1$

A3. $\mathrm{x} \vdash\left(\left(\mathrm{x}^{\dagger} \vdash \mathrm{y}\right) \vdash \mathrm{x}\right)=\mathrm{y} \vdash \mathrm{x}$

A4. $\mathrm{x}^{\dagger} \vdash 1=\mathrm{x}^{\dagger}$.

A5. $\mathrm{x} \vdash\left(\mathrm{x}^{\dagger} \vdash(\mathrm{y} \vdash 1)\right)=\mathrm{y} \vdash 1$.

A6. $(\mathrm{x} \vdash 1) \vdash \mathrm{y}=\mathrm{x} \vdash \mathrm{y}$.

We first show that $G$ must satisfy $A 1-A 6$ then show that $\vdash$ is associative.

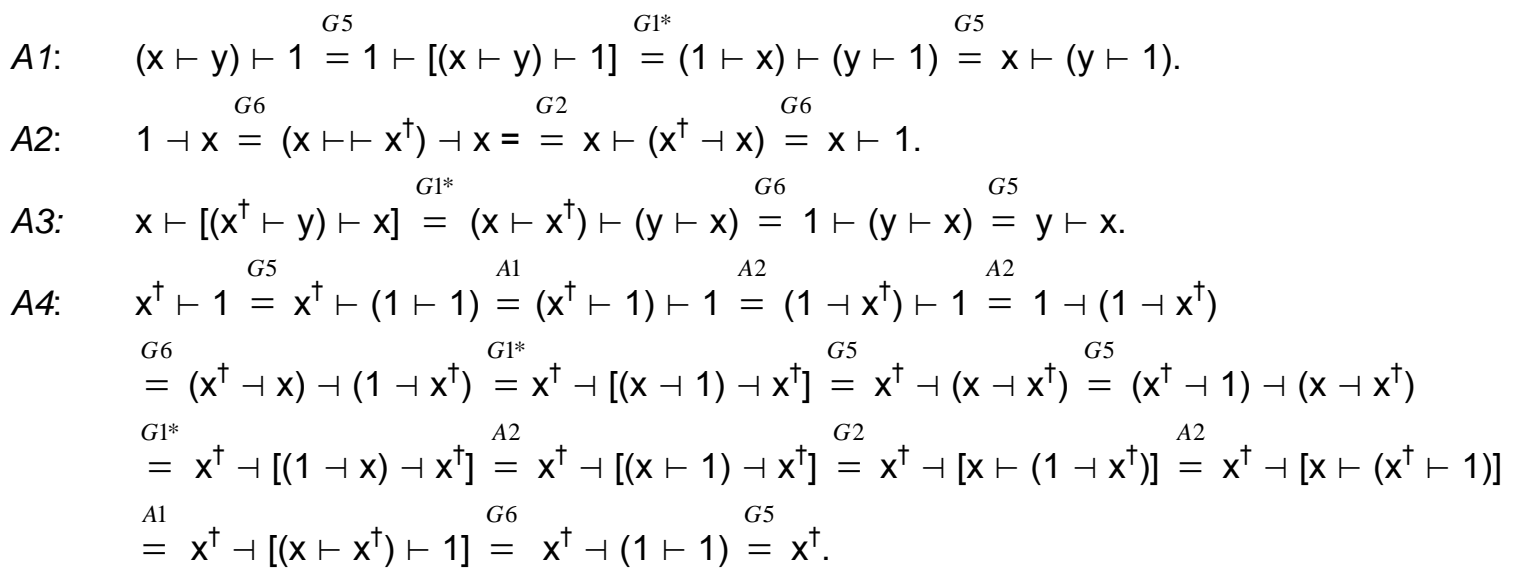


A5: $\quad \mathrm{x} \vdash\left[\mathrm{x}^{\dagger} \vdash(\mathrm{y} \vdash 1) \stackrel{A 2}{=} \mathrm{x} \vdash\left[\mathrm{x}^{\dagger} \vdash(1 \dashv \mathrm{y})\right] \stackrel{G 2}{=} \mathrm{x} \vdash\left[\left(\mathrm{x}^{\dagger} \vdash 1\right) \dashv \mathrm{y}\right] \stackrel{A 2}{=} \mathrm{x} \vdash\left[\left(1 \dashv \mathrm{x}^{\dagger}\right) \dashv \mathrm{y}\right]\right.$

$$
\begin{aligned}
& \stackrel{G 5}{=} \mathrm{x} \vdash\left[\left(1 \dashv \mathrm{x}^{\dagger}\right) \dashv(\mathrm{y} \dashv 1)\right] \stackrel{G 1^{*}}{=} \mathrm{x} \vdash\left[1 \dashv\left(\mathrm{x}^{\dagger} \dashv \mathrm{y}\right) \dashv 1\right] \stackrel{G 5}{=} \mathrm{x} \vdash\left[1 \dashv\left(\mathrm{x}^{\dagger} \dashv \mathrm{y}\right)\right] \\
& \text { A2 } \mathrm{x} \vdash\left[\left(\mathrm{x}^{\dagger} \dashv \mathrm{y}\right) \vdash 1\right]=\left[\mathrm{x} \vdash\left(\mathrm{x}^{\dagger} \dashv \mathrm{y}\right)\right] \stackrel{G 2}{=}\left[\left(\mathrm{x} \vdash \mathrm{x}^{\dagger}\right) \dashv \mathrm{y}\right] \vdash 1 \stackrel{G 5}{=}(1 \dashv \mathrm{y}) \vdash 1 \\
& \stackrel{A 2}{=}(\mathrm{y} \vdash 1) \vdash 1 \stackrel{A 1}{=} \mathrm{y} \vdash(1 \vdash 1) \stackrel{G 5}{=} \mathrm{y} \vdash 1 .
\end{aligned}
$$

The following lengthy operations show that $\mathrm{G}$ satisfies $A 6$.

$$
\begin{aligned}
& \text { A6: } \quad(\mathrm{x} \vdash 1) \vdash \mathrm{y} \stackrel{A 3}{=} \mathrm{y} \vdash\left(\left(\mathrm{y}^{\dagger} \vdash(\mathrm{x} \vdash 1)\right) \vdash \mathrm{y}\right) \stackrel{A 1}{=} \mathrm{y} \vdash\left(\left(\left(\mathrm{y}^{\dagger} \vdash \mathrm{x}\right) \vdash 1\right) \vdash \mathrm{y}\right) \\
& \stackrel{G 1^{*}}{=}\left(\mathrm{y} \vdash\left(\mathrm{y}^{\dagger} \vdash \mathrm{x}\right)\right) \vdash(1 \vdash \mathrm{y}) \stackrel{G 5}{=}\left(\mathrm{y} \vdash\left(\mathrm{y}^{\dagger} \vdash \mathrm{x}\right)\right) \vdash \mathrm{y} \stackrel{A 3}{=} \mathrm{y} \vdash\left(\left(\mathrm{y}^{\dagger} \vdash\left(\mathrm{y} \vdash\left(\mathrm{y}^{\dagger} \vdash \mathrm{x}\right)\right)\right) \vdash \mathrm{y}\right) \\
& \stackrel{A 4}{=} \mathrm{y} \vdash\left(\left(\left(\mathrm{y}^{\dagger} \vdash 1\right) \vdash\left(\mathrm{y} \vdash\left(\mathrm{y}^{\dagger} \vdash \mathrm{x}\right)\right)\right) \vdash \mathrm{y}\right) \\
& \text { A5 } \\
& =\mathrm{y} \vdash\left(\left(\left(\left(\mathrm{y}^{\dagger} \vdash \mathrm{x}\right) \vdash\left(\left(\mathrm{y}^{\dagger} \vdash \mathrm{x}\right)^{\dagger} \vdash\left(\mathrm{y}^{\dagger} \vdash 1\right)\right)\right) \vdash\left(\left(\mathrm{y} \vdash\left(\mathrm{y}^{\dagger} \vdash 1\right)\right)\right) \vdash \mathrm{y}\right)\right. \\
& \stackrel{A 4}{=} \mathrm{y} \vdash\left(\left(\left(\left(\mathrm{y}^{\dagger} \vdash \mathrm{x}\right) \vdash\left(\left(\mathrm{y}^{\dagger} \vdash \mathrm{x}\right)^{\dagger} \vdash \mathrm{y}^{\dagger}\right)\right) \vdash\left(\mathrm{y} \mathrm{y}^{\dagger}\left(\mathrm{y}^{\dagger} \vdash \mathrm{x}\right)\right)\right) \vdash \mathrm{y}\right) \\
& \text { G1* } \\
& =\mathrm{y} \vdash\left(\left(\left(\mathrm{y}^{\dagger} \vdash \mathrm{x}\right) \vdash\left(\left(\left(\left(\mathrm{y}^{\dagger} \vdash \mathrm{x}\right)^{\dagger} \vdash \mathrm{y}^{\dagger}\right) \vdash \mathrm{y}\right) \vdash\left(\mathrm{y}^{\dagger} \vdash \mathrm{x}\right)\right)\right) \vdash \mathrm{y}\right) \\
& =\mathrm{y} \vdash\left(\left(\left(\mathrm{y}^{\dagger} \vdash \mathrm{x}\right) \vdash\left(\left(\left(\left(\mathrm{y}^{\dagger} \vdash \mathrm{x}\right)^{\dagger} \vdash\left(1 \vdash \mathrm{y}^{\dagger}\right)\right) \vdash \mathrm{y}\right) \vdash\left(\mathrm{y}^{\dagger} \vdash \mathrm{x}\right)\right)\right) \vdash \mathrm{y}\right) \\
& =\mathrm{A} 4 \mathrm{y} \vdash\left(\left(\left(\mathrm{y}^{\dagger} \vdash \mathrm{x}\right) \vdash\left(\left(\left(\left(\mathrm{y}^{\dagger} \vdash \mathrm{x}\right)^{\dagger} \vdash\left(1 \vdash\left(\mathrm{y}^{\dagger} \vdash 1\right)\right)\right) \vdash \mathrm{y}\right) \vdash\left(\mathrm{y}^{\dagger} \vdash \mathrm{x}\right)\right)\right) \vdash \mathrm{y}\right) \\
& \left.\stackrel{A 2}{=} \mathrm{y} \vdash\left(\left(\left(y^{\dagger} \vdash x\right) \vdash\left(\left(\left(y^{\dagger} \vdash x\right)^{\dagger} \vdash\left(1 \vdash\left(1 \dashv y^{\dagger}\right)\right)\right) \vdash y\right) \vdash\left(y^{\dagger} \vdash x\right)\right)\right) \vdash y\right) \\
& \text { G2 } \\
& =\mathrm{y} \vdash\left(\left(\left(\mathrm{y}^{\dagger} \vdash \mathrm{x}\right) \vdash\left(\left(\left(\left(\mathrm{y}^{\dagger} \vdash \mathrm{x}\right)^{\dagger} \vdash\left((1 \vdash 1) \dashv \mathrm{y}^{\dagger}\right)\right) \vdash \mathrm{y}\right) \vdash\left(\mathrm{y}^{\dagger} \vdash \mathrm{x}\right)\right)\right) \vdash \mathrm{y}\right) \\
& \text { G2 } \\
& \left.=\mathrm{y} \vdash\left(\left(\left(y^{\dagger} \vdash \mathrm{x}\right) \vdash\left(\left(\left(\left(\mathrm{y}^{\dagger} \vdash \mathrm{x}\right)^{\dagger} \vdash(1 \vdash 1)\right) \dashv \mathrm{y}^{\dagger}\right) \vdash \mathrm{y}\right) \vdash\left(\mathrm{y}^{\dagger} \vdash \mathrm{x}\right)\right)\right) \vdash \mathrm{y}\right) \\
& \left.\stackrel{A 1}{=} \mathrm{y} \vdash\left(\left(\left(y^{\dagger} \vdash \mathrm{x}\right) \vdash\left(\left(\left(\left(\left(y^{\dagger} \vdash \mathrm{x}\right)^{\dagger} \vdash 1\right) \vdash 1\right) \dashv \mathrm{y}^{\dagger}\right) \vdash \mathrm{y}\right) \vdash\left(\mathrm{y}^{\dagger} \vdash \mathrm{x}\right)\right)\right) \vdash \mathrm{y}\right) \\
& \text { G2 } \\
& \left.=\mathrm{y} \vdash\left(\left(\left(\mathrm{y}^{\dagger} \vdash \mathrm{x}\right) \vdash\left(\left(\left(\left(\mathrm{y}^{\dagger} \vdash \mathrm{x}\right)^{\dagger} \vdash 1\right) \vdash\left(1 \dashv \mathrm{y}^{\dagger}\right)\right) \vdash \mathrm{y}\right) \vdash\left(\mathrm{y}^{\dagger} \vdash \mathrm{x}\right)\right)\right) \vdash \mathrm{y}\right) \\
& \text { A2 } \\
& \left.=\mathrm{y} \vdash\left(\left(\left(\mathrm{y}^{\dagger} \vdash \mathrm{x}\right) \vdash\left(\left(\left(\left(\mathrm{y}^{\dagger} \vdash \mathrm{x}\right)^{\dagger} \vdash 1\right) \vdash\left(\mathrm{y}^{\dagger} \vdash 1\right)\right) \vdash \mathrm{y}\right) \vdash\left(\mathrm{y}^{\dagger} \vdash \mathrm{x}\right)\right)\right) \vdash \mathrm{y}\right) \\
& \left.\stackrel{A 4}{=} \mathrm{y} \vdash\left(\left(\left(y^{\dagger} \vdash \mathrm{x}\right) \vdash\left(\left(\left(\left(y^{\dagger} \vdash \mathrm{x}\right)^{\dagger} \vdash 1\right) \vdash \mathrm{y}^{\dagger}\right) \vdash \mathrm{y}\right) \vdash\left(\mathrm{y}^{\dagger} \vdash \mathrm{x}\right)\right)\right) \vdash \mathrm{y}\right) \\
& \stackrel{A 3}{=} \mathrm{y} \vdash\left(\left(\left(y^{\dagger} \vdash \mathrm{x}\right) \vdash\left(\left(\mathrm{y} \vdash\left(\left(\mathrm{y}^{\dagger} \vdash\left(\left(\left(\mathrm{y}^{\dagger} \vdash \mathrm{x}\right)^{\dagger} \vdash 1\right) \vdash \mathrm{y}^{\dagger}\right)\right) \vdash \mathrm{y}\right)\right) \vdash\left(\mathrm{y}^{\dagger} \vdash \mathrm{x}\right)\right)\right) \vdash \mathrm{y}\right) \\
& \stackrel{A 4}{=} \mathrm{y} \vdash\left(\left(\left(\mathrm{y}^{\dagger} \vdash \mathrm{x}\right) \vdash\left(\left(\mathrm{y} \vdash\left(\left(\mathrm{y}^{\dagger} \vdash\left(\left(\left(\mathrm{y}^{\dagger} \vdash \mathrm{x}\right)^{\dagger} \vdash 1\right) \vdash\left(\mathrm{y}^{\dagger} \vdash 1\right)\right)\right) \vdash \mathrm{y}\right) \vdash\left(\mathrm{y}^{\dagger} \vdash \mathrm{x}\right)\right)\right) \vdash \mathrm{y}\right)\right. \\
& \stackrel{A 2}{=} \mathrm{y} \vdash\left(\left(\left(\mathrm{y}^{\dagger} \vdash \mathrm{x}\right) \vdash\left(\left(\mathrm{y} \vdash\left(\left(\mathrm{y}^{\dagger} \vdash\left(\left(\left(\mathrm{y}^{\dagger} \vdash \mathrm{x}\right)^{\dagger} \vdash 1\right) \vdash\left(1 \dashv \mathrm{y}^{\dagger}\right)\right)\right) \vdash \mathrm{y}\right) \vdash\left(\mathrm{y}^{\dagger} \vdash \mathrm{x}\right)\right)\right) \vdash \mathrm{y}\right)\right. \\
& =\mathrm{g} \vdash\left(\left(\left(\mathrm{y}^{\dagger} \vdash \mathrm{x}\right) \vdash\left(\left(\mathrm{y} \vdash\left(\left(\mathrm{y}^{\dagger} \vdash\left(\left(\left(\left(\mathrm{y}^{\dagger} \vdash \mathrm{x}\right)^{\dagger} \vdash 1\right) \vdash 1\right) \dashv \mathrm{y}^{\dagger}\right)\right) \vdash \mathrm{y}\right)\right) \vdash\left(\mathrm{y}^{\dagger} \vdash \mathrm{x}\right)\right)\right) \vdash \mathrm{y}\right) \\
& \stackrel{G 2}{=} \mathrm{y} \vdash\left(\left(\left(\mathrm{y}^{\dagger} \vdash \mathrm{x}\right) \vdash\left(\left(\mathrm{y} \vdash\left(\left(\left(\mathrm{y}^{\dagger} \vdash\left(\left(\left(\mathrm{y}^{\dagger} \vdash \mathrm{x}\right)^{\dagger} \vdash 1\right) \vdash 1\right)\right) \dashv \mathrm{y}^{\dagger}\right) \vdash \mathrm{y}\right)\right) \vdash\left(\mathrm{y}^{\dagger} \vdash \mathrm{x}\right)\right)\right) \vdash \mathrm{y}\right) \\
& \left.\stackrel{A 1}{=} \mathrm{y} \vdash\left(\left(\left(y^{\dagger} \vdash x\right) \vdash\left(\left(y \vdash\left(\left(\left(y^{\dagger} \vdash\left(\left(y^{\dagger} \vdash x\right)^{\dagger} \vdash 1\right)\right) \vdash 1\right) \dashv y^{\dagger}\right) \dashv y\right)\right) \vdash\left(y^{\dagger} \vdash x\right)\right)\right) \vdash y\right)
\end{aligned}
$$




$$
\begin{aligned}
& G 2 \\
& =\mathrm{y} \vdash\left(\left(\left(\mathrm{y}^{\dagger} \vdash \mathrm{x}\right) \vdash\left(\left(\mathrm{y} \vdash\left(\left(\left(\mathrm{y}^{\dagger} \vdash\left(\left(\mathrm{y}^{\dagger} \vdash \mathrm{x}\right)^{\dagger} \vdash 1\right)\right) \vdash\left(1 \vdash \mathrm{y}^{\dagger}\right)\right) \vdash \mathrm{y}\right)\right) \vdash\left(\mathrm{y}^{\dagger} \vdash \mathrm{x}\right)\right)\right) \vdash \mathrm{y}\right) \\
& A 2 \\
& =\mathrm{y} \vdash\left(\left(\left(\mathrm{y}^{\dagger} \vdash \mathrm{x}\right) \vdash\left(\left(\mathrm{y} \vdash\left(\left(\left(\mathrm{y}^{\dagger} \vdash\left(\left(\mathrm{y}^{\dagger} \vdash \mathrm{x}\right)^{\dagger} \vdash 1\right)\right) \vdash\left(\mathrm{y}^{\dagger} \vdash 1\right)\right) \vdash \mathrm{y}\right)\right) \vdash\left(\mathrm{y}^{\dagger} \vdash \mathrm{x}\right)\right)\right) \vdash \mathrm{y}\right) \\
& A 4 \\
& =\mathrm{y} \vdash\left(\left(\left(\mathrm{y}^{\dagger} \vdash \mathrm{x}\right) \vdash\left(\left(\mathrm{y} \vdash\left(\left(\left(\mathrm{y}^{\dagger} \vdash\left(\left(\mathrm{y}^{\dagger} \vdash \mathrm{x}\right)^{\dagger} \vdash 1\right)\right) \vdash \mathrm{y}^{\dagger}\right) \vdash \mathrm{y}\right)\right) \vdash\left(\mathrm{y}^{\dagger} \vdash \mathrm{x}\right)\right)\right) \vdash \mathrm{y}\right) \\
& G 1^{*} \\
& =\mathrm{y} \vdash\left(\left(\left(\mathrm{y}^{\dagger} \vdash \mathrm{x}\right) \vdash\left(\left(\left(\mathrm{y} \vdash\left(\mathrm{y}^{\dagger} \vdash\left(\left(\mathrm{y}^{\dagger} \vdash \mathrm{x}\right)^{\dagger} \vdash 1\right)\right)\right) \vdash\left(\mathrm{y}^{\dagger} \vdash \mathrm{y}\right)\right) \vdash\left(\mathrm{y}^{\dagger} \vdash \mathrm{x}\right)\right)\right) \vdash \mathrm{y}\right) \\
& \text { A5 } \mathrm{y} \vdash\left(\left(\left(\mathrm{y}^{\dagger} \vdash \mathrm{x}\right) \vdash\left(\left(\left(\left(\mathrm{y}^{\dagger} \vdash \mathrm{x}\right)^{\dagger} \vdash\left(\mathrm{y}^{\dagger} \vdash \mathrm{y}\right)\right) \vdash\left(\mathrm{y}^{\dagger} \vdash \mathrm{x}\right)\right)\right) \vdash \mathrm{y}\right)\right. \\
& =\mathrm{A4} \\
& =\mathrm{y} \vdash\left(\left(\left(\mathrm{y}^{\dagger} \vdash \mathrm{x}\right) \vdash\left(\left(\left(\mathrm{y}^{\dagger} \vdash \mathrm{x}\right)^{\dagger} \vdash\left(\mathrm{y}^{\dagger} \vdash \mathrm{y}\right)\right) \vdash\left(\mathrm{y}^{\dagger} \vdash \mathrm{x}\right)\right)\right) \vdash \mathrm{y}\right) \\
& A 3 \\
& =\mathrm{y} \vdash\left(\left(\left(\mathrm{y}^{\dagger} \vdash \mathrm{y}\right) \vdash\left(\mathrm{y}^{\dagger} \vdash \mathrm{x}\right)\right) \vdash \mathrm{y}\right) \stackrel{G 1^{*}}{=}\left(\mathrm{y} \vdash\left(\mathrm{y}^{\dagger} \vdash \mathrm{y}\right)\right) \vdash\left(\left(\mathrm{y}^{\dagger} \vdash \mathrm{y}\right)\right) \vdash\left(\left(\mathrm{y}^{\dagger} \vdash \mathrm{x}\right) \vdash \mathrm{y}\right) \\
& A 4 \\
& =\left(\mathrm{y} \vdash\left(\left(\mathrm{y}^{\dagger} \vdash 1\right) \vdash \mathrm{y}\right)\right) \vdash\left(\left(\mathrm{y}^{\dagger} \vdash \mathrm{x}\right) \vdash \mathrm{y}\right)=(1 \vdash \mathrm{y}) \vdash\left(\left(\mathrm{y}^{\dagger} \vdash \mathrm{x}\right) \vdash \mathrm{y}\right) \\
& G 5 \\
& =\mathrm{y} \vdash\left(\left(\mathrm{y}^{\dagger} \vdash \mathrm{x}\right) \vdash \mathrm{y}\right)=\mathrm{A} \vdash \mathrm{y} .
\end{aligned}
$$

We now show that $\vdash$ is associative.

$$
\begin{aligned}
& \mathrm{x} \vdash(\mathrm{y} \vdash \mathrm{z}) \\
& \text { A6 } \\
& =(\mathrm{x} \vdash 1) \vdash(\mathrm{y} \vdash \mathrm{z}) \\
& \text { A6 } \\
& =(\mathrm{x} \vdash 1) \vdash((\mathrm{y} \vdash 1) \vdash \mathrm{z}) \\
& \stackrel{A 5}{=}\left(\mathrm{z} \vdash\left(\mathrm{z}^{\dagger} \vdash(\mathrm{x} \vdash 1)\right) \vdash((\mathrm{y} \vdash 1) \vdash \mathrm{z})\right. \\
& \text { G1* } \\
& =\mathrm{z} \vdash\left(\left(\left(z^{\dagger} \vdash(x \vdash 1)\right) \vdash(y \vdash 1)\right) \vdash z\right) \\
& \text { A2 } \\
& =\mathrm{z} \vdash\left(\left(\left(\mathrm{z}^{\dagger} \vdash(\mathrm{x} \vdash 1)\right) \vdash(1 \dashv \mathrm{y})\right) \vdash \mathrm{z}\right) \\
& \text { G2 } \\
& =\mathrm{z} \vdash\left(\left(\left(\mathrm{z}^{\dagger} \vdash(\mathrm{x} \vdash 1)\right) \dashv \mathrm{y}\right) \vdash \mathrm{z}\right) \\
& \text { A1 } \\
& =\mathrm{z} \vdash\left(\left(\left(z^{\dagger} \vdash((x \vdash 1) \vdash 1)\right) \dashv y\right) \vdash z\right) \\
& \text { G2 } \\
& =\mathrm{z} \vdash\left(\left(z^{\dagger}(((x \vdash 1) \vdash 1) \dashv y)\right) \vdash z\right) \\
& G 2 \\
& =\mathrm{z} \vdash\left(\left(\mathrm{z}^{\dagger} \vdash((\mathrm{x} \vdash 1) \vdash(1 \dashv \mathrm{y}))\right) \vdash \mathrm{z}\right) \\
& \text { A2 } \\
& =\mathrm{z} \vdash\left(\left(\mathrm{z}^{\dagger} \vdash((\mathrm{x} \vdash 1) \vdash(\mathrm{y} \vdash 1))\right) \vdash \mathrm{z}\right) \\
& \text { A3 } \\
& =((x \vdash 1) \vdash(y \vdash 1)) \vdash z \\
& \stackrel{A 2}{=}((x \vdash 1) \vdash(1 \dashv y)) \vdash z \\
& \stackrel{G 2}{=}(((x \vdash 1) \vdash 1) \dashv \mathrm{y}) \vdash \mathrm{z} \\
& =((x \vdash(1 \vdash 1)) \dashv y) \vdash z
\end{aligned}
$$

$$
\begin{aligned}
& G 2 \\
& =(x \vdash((1 \vdash 1) \dashv y)) \vdash z \\
& G 2 \\
& =(x \vdash(1 \vdash(1 \dashv y))) \vdash z \\
& A 2 \\
& =(x \vdash(1 \vdash(y \vdash 1))) \vdash z \\
& G 5 \\
& =(x \vdash(y \vdash 1) \vdash z \\
& A 1 \\
& =((x \vdash y) \vdash \vdash 1) \vdash z \\
& A 6 \\
& =(x \vdash y) \vdash z .
\end{aligned}
$$

\section{COMMUTING PAIRS UNDER $\vdash$}

\section{a. Example}

Suppose $G$ is a digroup that is not a group, and further suppose that $G$ has distinct elements $a$ and $b$ such that $a \vdash b=$ $b \vdash a$ and $a \vdash b \neq a \dashv b$. We will show that the order of $G$ must be composite, and further, that the set of pairs that commute under $\vdash$ including those of the form $(x, x)$ is a sub-right group of $\mathrm{G} \times \mathrm{G}$.

The following is the smallest such digroup of odd order found by the computer program Mace 4.

Let $G=\mathbb{Z}_{15}$. We define $\vdash$ and $\dashv$ as follows: 


\begin{tabular}{|c|c|c|c|c|c|c|c|c|c|c|c|c|c|c|c|}
\hline$\vdash$ & 0 & 1 & 2 & 3 & 4 & 5 & 6 & 7 & 8 & 9 & 10 & 11 & 12 & 13 & 14 \\
\hline 0 & 11 & 2 & 3 & 1 & 7 & 10 & 0 & 8 & 4 & 5 & 9 & 6 & 13 & 14 & 12 \\
\hline 1 & 0 & 1 & 2 & 3 & 4 & 5 & 6 & 7 & 8 & 9 & 10 & 11 & 12 & 13 & 14 \\
\hline 2 & 11 & 2 & 3 & 1 & 7 & 10 & 0 & 8 & 4 & 5 & 9 & 6 & 13 & 14 & 12 \\
\hline 3 & 6 & 3 & 1 & 2 & 8 & 9 & 11 & 4 & 7 & 10 & 5 & 0 & 14 & 12 & 13 \\
\hline 4 & 0 & 1 & 2 & 3 & 4 & 5 & 6 & 7 & 8 & 9 & 10 & 11 & 12 & 13 & 14 \\
\hline 5 & 6 & 3 & 1 & 2 & 8 & 9 & 11 & 4 & 7 & 10 & 5 & 0 & 14 & 12 & 13 \\
\hline 6 & 0 & 1 & 2 & 3 & 4 & 5 & 6 & 7 & 8 & 9 & 10 & 11 & 12 & 13 & 14 \\
\hline 7 & 11 & 2 & 3 & 1 & 7 & 10 & 0 & 8 & 4 & 5 & 9 & 6 & 13 & 14 & 12 \\
\hline 8 & 6 & 3 & 1 & 2 & 8 & 9 & 11 & 4 & 7 & 10 & 5 & 0 & 14 & 12 & 13 \\
\hline 9 & 11 & 2 & 3 & 1 & 7 & 10 & 0 & 8 & 4 & 5 & 9 & 6 & 13 & 14 & 12 \\
\hline 10 & 0 & 1 & 2 & 3 & 4 & 5 & 6 & 7 & 8 & 9 & 10 & 11 & 12 & 13 & 14 \\
\hline 11 & 6 & 3 & 1 & 2 & 8 & 9 & 11 & 4 & 7 & 10 & 5 & 0 & 14 & 12 & 13 \\
\hline 12 & 0 & 1 & 2 & 3 & 4 & 5 & 6 & 7 & 8 & 9 & 10 & 11 & 12 & 13 & 14 \\
\hline 13 & 11 & 2 & 3 & 1 & 7 & 10 & 0 & 8 & 4 & 5 & 9 & 6 & 13 & 14 & 12 \\
\hline 14 & 6 & 3 & 1 & 2 & 8 & 9 & 11 & 4 & 7 & 10 & 5 & 0 & 14 & 12 & 13 \\
\hline$\dashv$ & 0 & 1 & 2 & 3 & 4 & 5 & 6 & 7 & 8 & 9 & 10 & 11 & 12 & 13 & 14 \\
\hline 0 & 5 & 0 & 5 & 4 & 0 & 4 & 0 & 5 & 4 & 5 & 0 & 4 & 0 & 5 & 4 \\
\hline 1 & 2 & 1 & 2 & 3 & 1 & 3 & 1 & 2 & 3 & 2 & 1 & 3 & 1 & 2 & 3 \\
\hline 2 & 3 & 2 & 3 & 1 & 2 & 1 & 2 & 3 & 1 & 3 & 2 & 1 & 2 & 3 & 1 \\
\hline 3 & 1 & 3 & 1 & 2 & 3 & 2 & 3 & 1 & 2 & 1 & 3 & 2 & 3 & 1 & 2 \\
\hline 4 & 0 & 4 & 0 & 5 & 4 & 5 & 4 & 0 & 5 & 0 & 4 & 5 & 4 & 0 & 5 \\
\hline 5 & 4 & 5 & 4 & 0 & 5 & 0 & 5 & 4 & 0 & 4 & 5 & 0 & 5 & 4 & 0 \\
\hline 6 & 9 & 6 & 9 & 8 & 6 & 8 & 6 & 9 & 8 & 9 & 6 & 8 & 6 & 9 & 8 \\
\hline 7 & 11 & 7 & 11 & 10 & 7 & 10 & 7 & 11 & 10 & 11 & 7 & 10 & 7 & 11 & 10 \\
\hline 8 & 6 & 8 & 6 & 9 & 8 & 9 & 8 & 6 & 9 & 6 & 8 & 9 & 8 & 6 & 9 \\
\hline 9 & 8 & 9 & 8 & 6 & 9 & 6 & 9 & 8 & 6 & 8 & 9 & 6 & 9 & 8 & 6 \\
\hline 10 & 7 & 10 & 7 & 11 & 10 & 11 & 10 & 7 & 11 & 7 & 10 & 11 & 10 & 7 & 11 \\
\hline 11 & 10 & 11 & 10 & 7 & 11 & 7 & 11 & 10 & 7 & 10 & 11 & 7 & 11 & 10 & 7 \\
\hline 12 & 13 & 12 & 13 & 14 & 12 & 14 & 12 & 13 & 14 & 13 & 12 & 14 & 12 & 13 & 14 \\
\hline 13 & 14 & 13 & 14 & 12 & 13 & 12 & 13 & 14 & 12 & 14 & 13 & 12 & 13 & 14 & 12 \\
\hline 14 & 12 & 14 & 12 & 13 & 14 & 13 & 14 & 12 & 13 & 12 & 14 & 13 & 14 & 12 & 13 \\
\hline
\end{tabular}

Here $9 \vdash 5=5 \vdash 9=10 \neq 6=9 \dashv 5$.

\section{b. The Order of $G$}

We first collect some basic definitions and results about right groups that we will need to prove our results (Lemma 1). Note that left groups have results corresponding to those listed for right groups.

Lemma 1. [2] Let $(G, \vdash)$ be a right group, let $E=\{e \in G \mid e \vdash x=x\}$, and let $J=\left\{x^{-1} \mid x \in\right.$ $\mathrm{G}$ \}.

1. $(x \vdash y)^{-1}=y^{-1} \vdash x^{-1}$ for all $x, y \in G$.

2. $x \vdash 1=\left(x^{-1}\right)^{-1}$ for all $x \in G$.

3. $E$ is a right zero semigroup.
4. $\mathrm{J}$ is a group.

5. $\mathrm{G}=\mathrm{J} \vdash \mathrm{E} \cong(\mathrm{J} \times \mathrm{E}, \vdash)$.

6. $G$ is a group if and only if $\vdash=\dashv$ if and only if $E=\{1\}$.

Theorem 3. If $G$ is a digroup with $a, b \in G$ satisfying $a \vdash b=b \vdash a$ and $a \vdash b \neq a \dashv b$, the $|G|$ is composite.

Proof. Let $(G, \vdash, \dashv)$ be a digroup and suppose $|G|=p$ for some prime $p$. From Lemma 1 we know that $\mathrm{G} \cong(\mathrm{J} \times \mathrm{E}, \vdash)$. Therefore $|\mathrm{E}|=1$ or $|\mathrm{E}|=p$. Suppose $|\mathrm{E}|$ $=1$. Then by Lemma $1, \vdash=\dashv$ and therefore 
$a \vdash b=a \dashv b$, a contradiction. Suppose $|E|$ $=p$. Then $\forall_{g_{i}}, g_{j} \in \mathrm{G}, g_{i} \vdash g_{j}=g_{j}$. Therefore, as illustrated by the Cayley table below, there are no commutative pairs in $(\mathrm{G}, \vdash)$.

\begin{tabular}{|c|c|c|c|c|c|}
\hline$\vdash$ & $g_{1}$ & $g_{2}$ & $\mathrm{~g}_{3}$ & $\ldots$ & $g_{p}$ \\
\hline$g_{1}$ & $g_{1}$ & $g_{2}$ & $g_{3}$ & $\ldots$ & $g_{p}$ \\
\hline$g_{2}$ & $g_{1}$ & $g_{2}$ & $g_{3}$ & $\ldots$ & $g_{p}$ \\
\hline$g_{3}$ & $g_{1}$ & $g_{2}$ & $g_{3}$ & $\ldots$ & $g_{p}$ \\
\hline$\vdots$ & $\vdots$ & $\vdots$ & $\vdots$ & $\vdots$ & $\vdots$ \\
\hline$g_{p}$ & $g_{1}$ & $g_{2}$ & $g_{3}$ & $\ldots$ & $g_{p}$ \\
\hline
\end{tabular}

c. Commuting Pairs Under $\vdash$

Here we list all the pairs from Example 1 that commute under $\vdash$, excluding pairs of the form $(x, x)$, and we also show $J$ $\vdash \mathrm{E}$. Here $\mathrm{J}=\{1,2,3\} \cong \mathbb{Z}_{3}$ and $\mathrm{E}=\{1,4$, $6,10,12\}$.

\section{Commutative Pairs Under $\vdash$}

\begin{tabular}{ccc}
\hline$(1,2)$ & $(1,3)$ & $(2,3)$ \\
$(4,7)$ & $(4,8)$ & $(7,8)$ \\
$(6,0)$ & $(6,11)$ & $(0,11)$ \\
$(10,5)$ & $(10,9)$ & $(5,9)$ \\
$(12,13)$ & $(12,14)$ & $(13,14)$ \\
& $\mathrm{J} \vdash \mathrm{E}$ & \\
$3 \vdash 1=3$ & $2 \vdash 1=2$ & $1 \vdash 1=1$ \\
$3 \vdash 4=8$ & $2 \vdash 4=7$ & $1 \vdash 4=4$ \\
$3 \vdash 6=11$ & $2 \vdash 6=0$ & $1 \vdash 6=6$ \\
$3 \vdash 10=5$ & $2 \vdash 10=9$ & $1 \vdash 10=10$ \\
$3 \vdash 12=14$ & $2 \vdash 12=13$ & $1 \vdash 12=12$
\end{tabular}

From these tables we notice two things. First, going across a row of $\mathrm{J} \vdash \mathrm{E}$, all elements commute, e.g., 8 and 7 commute,
4 and 7 commute, and 4 and 8 commute. Second, going down a column of $\mathrm{J} \vdash \mathrm{E}$, no elements commute, either with elements of their own column or with elements of other columns not also in the same row, e.g., 11 and 7 do not commute. We can generalize these observations as follows.

For each of the following lemmas, let $\mathrm{j}_{\mathrm{x}} \in \mathrm{J}$ and let $\mathrm{e}_{\mathrm{x}} \in \mathrm{E}$.

Lemma 2. If $\mathrm{J}$ is abelian and $e$ is a fixed element of $E$, then all pairs of the form $\left(\mathrm{j}_{\mathrm{k}} \vdash\right.$ $\left.\mathrm{e}, \mathrm{j}_{1} \vdash \mathrm{e}\right)$ commute.

Proof. Assume $\mathrm{J}$ is abelian and choose $e$ a fixed element of $\mathrm{E}$. Since $\mathrm{J} \leq \mathrm{G}, \mathrm{j}_{\mathrm{k}} \vdash \mathrm{j}_{\mathrm{l}}=\mathrm{j}_{\mathrm{kl}}$ $\in \mathrm{J}$. Therefore $\left(\mathrm{j}_{\mathrm{k}} \vdash \mathrm{e}\right) \vdash\left(\mathrm{j}_{\mathrm{l}} \vdash \mathrm{e}\right)=\mathrm{j}_{\mathrm{k}} \vdash(\mathrm{e} \vdash$ $\left.\mathrm{j}_{\mathrm{l}}\right) \vdash \mathrm{e}=\mathrm{j}_{\mathrm{kl}} \vdash \mathrm{e}=\mathrm{j}_{\mathrm{l}} \vdash \mathrm{e}=\left(\mathrm{j}_{\mathrm{l}} \vdash \mathrm{e}\right) \vdash\left(\mathrm{j}_{\mathrm{k}} \vdash \mathrm{e}\right)$.

Lemma 3. Define $\sim$ by $a \sim b$ if $a=j_{k} \vdash e$ and $\mathrm{b}=\mathrm{j}_{1} \vdash \mathrm{e}$ for the same e. Clearly $\sim$ is an equivalence relation.

Lemma 4. No pairs of the form $\left(\mathrm{j}_{\mathrm{k}} \vdash \mathrm{e}_{1}, \mathrm{j}_{1} \vdash\right.$ $\mathrm{e}_{2}$ ) commute, where $\mathrm{e}_{1} \neq \mathrm{e}_{2}$.

Proof. $\left(\mathrm{j}_{\mathrm{k}} \vdash \mathrm{e}_{1}\right) \vdash\left(\mathrm{j}_{1} \vdash \mathrm{e}_{2}\right)=\mathrm{j}_{\mathrm{kl}} \vdash \mathrm{e}_{2}$, while $\left(\mathrm{j}_{1}\right.$ $\left.\vdash \mathrm{e}_{2}\right) \vdash\left(\mathrm{j}_{\mathrm{k}} \vdash \mathrm{e}_{1}\right)=\mathrm{j}_{\mathrm{k}} \vdash \mathrm{e}_{1}$. From Lemma 3, we know that $\mathrm{j}_{\mathrm{kl}} \vdash \mathrm{e}_{2} \neq \mathrm{j}_{\mathrm{l} \mathrm{k}} \vdash \mathrm{e}_{1}$.

There is one further lemma that will be useful to us.

Lemma 5. $\exists a, b \in(G, \vdash, \dashv)$ satisfying $a$ $\vdash \mathrm{b}=\mathrm{b} \vdash \mathrm{a}$ if and only if $\mathrm{a}^{-1} \vdash \mathrm{b}^{-1}=\mathrm{b}^{-1} \vdash \mathrm{a}^{-1}$.

Proof. Let $\mathrm{G}$ be a digroup.

$\Rightarrow$ Let $a, b \in G$ such that $a \vdash b=b \vdash a$. Then $b^{-1} \vdash a^{-1}=(a \vdash b)^{-1}=(b \vdash a)^{-1}=a^{-1} \vdash$ $\mathrm{b}^{-1}$.

$\Leftarrow$ Let $a, b \in G$ such that $a^{-1} \vdash b^{-1}=b^{-1} \vdash a^{-1}$. Then $(b \vdash a)^{-1}=a^{-1} \vdash b^{-1}=b^{-1} \vdash a^{-1}=(a \vdash$ b) $)^{-1}$.

Definition 2. Let $H=\{(a, b) \in(G \times G, \vdash) \mid a$ $\vdash \mathrm{b}=\mathrm{b} \vdash \mathrm{a}\}$. For all $(\mathrm{a}, \mathrm{b}),(\mathrm{c}, \mathrm{d}) \in \mathrm{H}$, 
define $(\mathrm{a}, \mathrm{b}) \vdash(\mathrm{c}, \mathrm{d})$ by $(\mathrm{a}, \mathrm{b}) \vdash(\mathrm{c}, \mathrm{d})=(\mathrm{a} \vdash \mathrm{c}$, $b \vdash d)$.

Theorem 4. If $\mathrm{J}$ is an abelian subgroup of a digroup $G$, then $H$ is a sub-right group of $(G$ $\times \mathrm{G}, \vdash)$.

Proof. Let $\mathrm{G}$ be a digroup. Clearly $(\mathrm{G} \times \mathrm{G}$, $\vdash)$ is a right group. Let $\mathrm{J}$ be an abelian subgroup of $\mathrm{G}$, and let $(a, b),(c, d) \in H$.

Closure: From Lemma 4 we know we can write $(\mathrm{a}, \mathrm{b})$ as $\left(\mathrm{j}_{\mathrm{a}} \vdash \mathrm{e}_{\mathrm{a}}, \mathrm{j}_{\mathrm{b}} \vdash \mathrm{e}_{\mathrm{a}}\right)$ and $(\mathrm{c}, \mathrm{d})$ as $\left(\mathrm{j}_{\mathrm{c}}\right.$ $\left.\vdash \mathrm{e}_{\mathrm{c}}, \mathrm{j}_{\mathrm{d}} \vdash \mathrm{e}_{\mathrm{c}}\right)$. Therefore $(\mathrm{a}, \mathrm{b}) \vdash(\mathrm{c}, \mathrm{d})=\left(\mathrm{j}_{\mathrm{a}} \vdash\right.$ $\left.\mathrm{e}_{\mathrm{a}} \vdash \mathrm{j}_{\mathrm{c}} \vdash \mathrm{e}_{\mathrm{c}}, \mathrm{j}_{\mathrm{b}} \vdash \mathrm{e}_{\mathrm{a}} \vdash \mathrm{j}_{\mathrm{d}} \vdash \mathrm{e}_{\mathrm{c}}\right)=\left(\mathrm{j}_{\mathrm{ac}} \vdash \mathrm{e}_{\mathrm{c}}, \mathrm{j}_{\mathrm{bd}}\right.$ $\vdash \mathrm{e}_{\mathrm{c}}$ ), which commutes since $\mathrm{J}$ is abelian. Therefore $\mathrm{H}$ is closed under $\vdash$.

Right Inverses: From Lemma 5, we know that $\left(a^{-1}, b^{-1}\right) \in H$. Obviously $(a, b) \vdash$ $\left(a^{-1}, b^{-1}\right)=(1,1) \in H$.

Left Identity: Clearly $(1,1) \vdash(a, b)=(a, b)$.

\section{ACKNOWLEDGEMENTS}

We would like to thank professor J. D. Phillips at Wabash College for advice. Also, thanks to Jacob Carson and Mollie Kerrigan for conversations and correspondence.

\section{REFERENCES}

1. J. L. Loday, Une version non commutative desalgébres de Lie: les algebras de Leibniz, Enseign. Math. 39 (1993) $269-293$.

2. Michael K. Kinyon, Leibniz algebras, Lie racks, and digroups, submitted for publication. Available at www.arXic:math.RA/0311396.

3. J. L. Loday, in: Dialgebras and Related Operands [Lecture Notes in Math. Series, 1763] (Springer, Berlin, 2001) 7 $-66$.

4. Raul Felipe, Generalized Loday algebras and digroups, Communicaciones del CIMAT, no. I-0401/21-01-2004.
5. Keqin Liu, A class of group-like objects, submitted for publication. Available at www.arXic:math.RA/0403509.

6. J. D. Phillips, A short Basis for the Variety of Digroups, Semigroup Forum OF1-OF5, 2004.

\section{Wabash College A LIBERAL ARTS COLLEGE FOR MEN}

\section{Wabash College is a private, independent, four-year liberal arts college for men, granting Bachelor of Arts degree.}

Wabash College is located in Crawfordsville, Indiana, a community of 14,000 located 45 miles northwest of Indianapolis and 150 miles southeast of Chicago. The 60-acre wooded campus contains 25 buildings predominantly of Georgian architecture. Caleb Mills taught the first class of Wabash students in 1833 in Forest Hall, located since 1965 at the north end of campus and now home to the Teacher Education Department. Built in 1836, Caleb Mills' House hosts various college functions. Also built in 1836, Hovey Cottage, home to the College's second faculty member Edmund O. Hovey, houses the Wabash Center for Teaching and Learning in Theology and Religion. The College dedicated the newly expanded and renovated Fine Arts Center in 1993. The Detchon Center for Modern Languages and International Studies, housed in an expanded and renovated campus landmark built in 1893, is a state-of-theart facility. During the Campaign for Leadership, Wabash built Hays Hall, the $\$ 30$ million home of the biology and chemistry departments; renovated Goodrich Hall, which is home to the mathematics and physics departments; built a \$2 million Malcolm X Institute for Black Studies; and built the $\$ 20$ million Allen Athletics and Recreation Center.

www.wabash.edu 


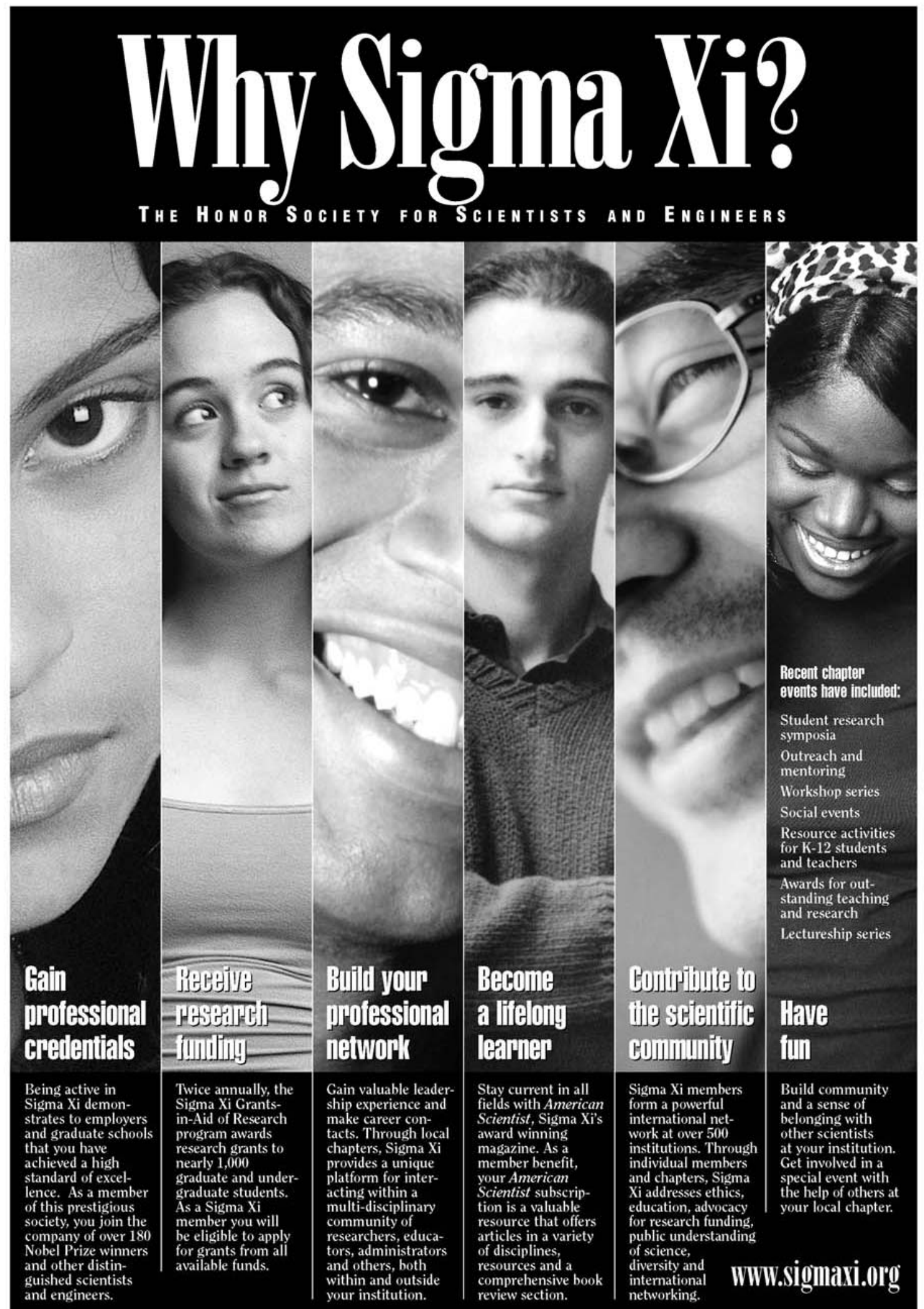

Sigma Xi, The Scientific Research Society • 98 Alexander Drive • P.O. Box 13975 • Research Triangle Park, NC 27708 • 818-549-4691 • 800-243-6534 